\title{
Compreendendo o Fenômeno do Cyberbullying
}

\author{
Guilherme Welter Wendt ${ }^{1}$ \\ Unit for School and Family Studies of the Goldsmiths College at University of London, \\ London, Grã-Bretanha \\ Carolina Saraiva de Macedo Lisboa \\ Programa de Pós-Graduação em Psicologia da Pontifícia Universidade Católica \\ do Rio Grande do Sul, Porto Alegre, Rio Grande do Sul, Brasil
}

\section{Resumo}

O subtipo específico de violência denominado de bullying constitui-se em um problema sério e recorrente nas instituições escolares atualmente, trazendo consequências negativas para o processo de desenvolvimento humano em curto, médio e longo prazo. Considerada uma questão de saúde pública, esta e outras formas de agressividade entre pares vêm atraindo significativa atenção por parte da mídia, dos pais, professores, cientistas e também dos responsáveis pela elaboração de políticas públicas. Entretanto, em um mundo "digital", o advento das novas tecnologias da informação e comunicação acaba também impulsionando a emergência de um novo tipo de bullying, que se situa no ciberespaço e se apoia nas ferramentas tecnológicas de interação, denominado cyberbullying. Assim, o presente artigo objetiva analisar e discutir os aspectos que tangenciam a ocorrência dessa forma eletrônica de comportamento agressivo e intencional, com o foco em questões conceituais que o diferenciam do bullying tradicional, impactos, fatores de risco e proteção deste processo. Para tal, serão apresentados e discutidos estudos sobre prevalência, características e consequências do cyberbullying selecionados nas seguintes bases de dados: Pepsic, Science Direct, BVS, PsychInfo, Scielo e PubMed. Tratou-se de uma revisão não-sistemática da literatura internacional e nacional sobre cyberbullying que permitiu uma reflexão acerca desse tipo de violência e possíveis caminhos para uma melhor compreensão de estratégias para o manejo das situações de cyberbullying na atualidade. A importância de pesquisas sobre esta temática no Brasil e América Latina é reforçada no sentido de aprofundar-se a compreensão e gerar subsídios para o desenvolvimento de intervenções focais e preventivas.

Palavras-chave: Cyberbullying, bullying, agressividade, adolescência, contextos virtuais.

\section{Understanding the Cyberbullying Phenomenon}

\section{Abstract}

The subtype of violence behavior named as bullying is a serious problem recurrent in schools nowadays, bringing negative short, medium and long term consequences to development process. Considered as a public health issue, this and other forms of peer aggression are attracting media, parents, teachers and scientists attention as well as attention from responsible for public policies. Therefore, in a digital world, the new information and communication technologies allow a new kind of bullying to take

Endereço para correspondência: Goldsmiths, University of London. Department of Psychology. Unit for School and Family Studies. The Bungalow Building, Room 4, Lewisham Way. London, Grã-Bretanha - SE14 6NW. E-mail: guilhermewwendt@gmail.com e lisboacaro@gmail.com.

Apoio: Coordenação de Aperfeiçoamento de Pessoal de Nível Superior (CAPES, processo BEX 893713-3) e International Society for the Study of Behavioural Development (ISSBD). 
place, a violence that situates in cyberspace and is supported by interactive technological tools, called cyberbullying. In this sense, the present paper aims to analyze and discuss aspects that are related to this electronic, intentional aggressive behavior, focusing in conceptual differences from bullying, impacts, risk and protective factors related to this process. To that, studies selected from Pepsic, Science Direct, BVS, PsychInfo, Scielo and PubMed about prevalence, characteristics and cyberbullying consequences are presented and discussed. The result was an unsystematic international and national literature revision toward cyberbullying that allowed an adequate reflection about this kind of violence and possible ways to a better comprehension and effective strategies to deal with cyberbullying nowadays. The importance of the researches about this topic in Brazil and Latin America is reinforced to provide a broader understanding about this process and to generate data that support the development of focus and preventive interventions.

Keywords: Cyberbullying, bullying, aggression, adolescence, virtual contexts.

\section{Compreendendo el Fenómeno del Ciberacoso}

\section{Resumen}

El tipo de violencia clasificado como bullying es un serio y recurrente problema en las escuelas en la actualidad, trayendo consecuencias negativas para El proceso de desarrollo humano, en curto, medio y largo plazo. Tenido como un tema de salud publica, esta y otras formas de agresividad entre pares están atrayendo significativa atención por parte de la media, de los padres, profesores, cientistas y también de los responsables por la elaboración de las políticas públicas. Sin embargo, en un mundo "digital”, las nuevas tecnologías de la información y comunicación también apuntan para la emergencia de un nuevo tipo de bullying, que se situa en el espacio virtual y apoyase en las herramientas tecnológicas de interacción, denominado de cyberbullying. Así, el presente artigo objetiva analisar y discutir los aspectos que influencian la ocurrencia de esa forma electrónica de comportamiento agresivo y intencional, con o foco en la definición conceptual que diferencia el ciberbullying del proceso de bullying, impactos, factores de riesgo e protección dese proceso. Para eso, serán presentados y discutidos estudios sobre prevalencia, características y consecuencias del cyberbullying seleccionados en las bases de datos: Pepsic, Science Direct, BVS, PsychInfo, Scielo e PubMed. Se ha realizado un revisión asistemático de la literatura internacional y nacional sobre el cyberbullying que ha permitido se pensar a cerca de ese tipo de violencia y los posibles caminos para una mejor comprensión de las estrategias para el manejo de las situaciones de cyberbullying en la actualidad. La importancia de investigaciones sobre esta temática en el Brasil y Latinoamérica es enfatizada en el sentido de se profundizar la comprensión e generar subsidios para El desarrollo de intervenciones focales y preventivas.

Palabras clave: Cyberbullying, bullying, agresión, adolescencia, contextos virtuales.

A agressividade humana pode ser definida como um comportamento que é motivado e que tem por objetivo causar algum tipo de sofrimento a outro indivíduo ou grupo (Anderson \& Bushman, 2002). Os atos de agressividade, inseridos em contexto cultural específico, repercutem negativamente nas mais distintas esferas da sociedade (família, trabalho, lazer, educação, etc). Nos últimos anos, a comunidade científica tem concentrado esforços para o entendimento mais aprofundado das diferentes manifestações da agressividade e de seus impactos na vida das pessoas (DeWall, Anderson, \& Bushman, 2011; Little, Henrich, Jones, \& Hawley, 2003). Quando se considera especificamente as crianças e os adolescentes, observa-se que a agressão pode resultar em consequências ainda mais graves. $\mathrm{Ou}$ seja, para além do impacto psicológico imediato da agressividade - considerando-se que no caso dos jovens pode não existir, ainda, maturidade 
suficiente para adequado manejo - os jovens podem interiorizar a noção de que somente através do uso da agressividade é que se torna possível a resolução de conflitos (Garaigordobil, 2011). De acordo com essa perspectiva de compreensão, a vivência de agressividade pode gerar comportamentos e crenças específicas acerca das relações interpessoais. Assim, indivíduos que agridem ou são agredidos podem aprender que esta é a forma de reagir em situações de estresse ou como um meio para atingir seus objetivos (Dodge, Bates, \& Pettit, 1990).

O bullying, palavra de origem estrangeira e sem tradução específica para a língua portuguesa, refere-se a um processo que pode ser circunscrito dentro do rol de comportamentos entendidos como agressivos ou violentos (Hong \& Espelage, 2012). Em geral, o uso do termo no contexto brasileiro alude a comportamentos de intimidação, violência e humilhação, embora o processo não se restrinja somente a essas ações (Wendt, Campos, \& Lisboa, 2010). Em termos conceituais, entende-se que o processo de bullying ocorre na medida em que uma pessoa ou um grupo busca, sistematicamente, excluir, intimidar, molestar ou maltratar outra pessoa ou mesmo um grupo de pessoas, levando a exclusão social (Olweus, 1993). Cabe mencionar também que se observa um demarcado desequilíbrio de poder nos casos de bullying, em que a vítima se encontra em uma situação com escassos ou nenhum recurso de defesa, além do caráter sistemático de agressão (Lisboa, Braga, \& Ebert, 2009). Além disso, o bullying pode ocorrer tanto de modo direto, através de atos envolvendo agressões físicas e ataques verbais, ou ainda de modo indireto e relacional, como nas situações de isolamento, chantagem, ameaças, difusão de rumores e fofocas, furtos, entre outros (Rigby, 2004).

Em relação à sua ocorrência, sabe-se que o processo de bullying é um fenômeno que se manifesta principalmente no contexto escolar, envolvendo crianças e adolescentes, e encontra-se presente em praticamente todas as culturas (Berger \& Lisboa, 2009). Do mesmo modo, o bullying ocorre no cotidiano tanto de escolas públicas e privadas como também em instituições localizadas no âmbito urbano e rural, sendo, portanto, um desafio recorrente em praticamente qualquer escola (Binsfeld \& Lisboa, 2010).

Para a Kandersteg Declaration Against Bullying in Children and Youth (2007), o bullying é um problema social que viola os direitos humanos básicos. Esta mesma declaração afirma que, em termos globais, cerca de $200 \mathrm{mi}-$ 1hões de crianças e adolescentes estão expostas ao fenômeno. No Brasil, conforme a investigação de Malta et al. (2010), os índices apontam que o percentual de vítimas de bullying atinge aproximadamente $30 \%$ dos alunos regularmente matriculados em escolas de ensino fundamental, sendo resultado de um processo complexo de interações recíprocas entre aspectos individuais e familiares das crianças e adolescentes, do contexto imediato e da sociedade em geral.

Por ser uma situação deletéria à saúde psicológica em curto, médio e longo prazo, tanto para os protagonistas do processo de bullying como também para os demais envolvidos, como professores, colegas e pais, o fenômeno tem recebido expressiva atenção por parte de estudiosos do campo da psicologia e também dos profissionais responsáveis pela implementação de políticas públicas de proteção aos direitos fundamentais da infância e adolescência (Binsfeld \& Lisboa, 2010; Hansen, Steenberg, Palic, \& Elklit, 2012; Smith, Smith, Osborn, \& Samara, 2008). Todavia, alguns pesquisadores vêm destacando um novo tipo de manifestação de agressividade entre pares, conhecido por cyberbullying (Law, Shapka, Hymel, Olson, \& Waterhouse, 2012; Shariff, 2011; Smith, 2012; Smith, Mahdavi, Carvalho, Fisher, Russel, \& Tippett, 2008), que apresenta impactos não menos severos que os do bullying e que ainda é muito pouco conhecido na sua especificidade (Garaigordobil, 2011; Sourander et al., 2010; Ybarra, Boyd, Korchmaros, \& Oppenheim, 2012). O fenômeno é considerado como um fato recente uma vez que as primeiras publicações sobre cyberbullying iniciaram há mais ou menos meia década atrás, sobretudo na Europa e nos Estados Unidos e, até a presente data, não se encontram estudos empíricos sobre esta temática no Brasil (Wendt et al., 2010). 
A variedade de investigações já conduzidas sobre a temática situa o cyberbullying como um fator de risco significativo para o desenvolvimento subsequente de crianças e adolescentes. Entendido como "um tipo de bullying que utiliza a tecnologia" (Shariff, 2011, p. 59), estudos reportam a associação desse tipo de violência com níveis elevados de ansiedade, uso e abuso de psicotrópicos, maior severidade de transtornos emocionais, como a depressão, ideias ou tentativas de suicídio, prejuízos na escola, dentre outros (Hinduja \& Patchin, 2010; Patchin \& Hinduja; 2010; Ybarra, 2004). Além disso, não somente as vítimas, como também os protagonistas dos atos de cyberbullying têm maiores chances de estabelecerem relações permeadas por conflitos, instabilidade e agressão (Shariff, 2011).

Logo, faz-se urgente e necessária uma maior compreensão acerca desse fenômeno para que seja possível o desenho de ações preventivas e interventivas eficazes. Conforme observam Wang, Nansel e Iannotti (2011), as pesquisas sobre o cyberbullying ainda encontram-se em uma etapa preliminar. Essa mesma constatação foi feita por Couvillon e Ilieva (2011), que sublinham a necessidade de esforços para o mapeamento dos mecanismos que favorecem o surgimento e a manutenção desse tipo de agressão entre pares.

Nesse sentido, a presente revisão crítica da literatura objetiva analisar e discutir os aspectos que tangenciam a ocorrência dessa forma eletrônica de comportamento agressivo e intencional, com o foco em questões conceituais que o diferenciam do bullying tradicional, impactos, fatores de risco e proteção deste processo. A revisão crítica que será apresentada assumiu um caráter não-sistemático, uma vez que não era objetivo quantificar os estudos e apresentar estatísticas mas sim de ampliar a compreensão e problematizar este novo tipo de comportamento agressivo entre pares e discutir aspectos que fazem a distinção entre o bullying praticado na escola daquele que ocorre no ciberespaço.

Estudos disponíveis nas bases de dados nacionais e internacionais (Scielo, Pepsic, BVS, PubMed, PsychInfo e ScienceDirect) foram recuperados através de descritores-alvo variados (cyberbullying, adolescentes, bullying virtual, por exemplo, bem como os respectivos equivalentes na língua inglesa e espanhola) e passaram então por uma análise qualitativa seletiva em relação à sua relevância e adequação aos propósitos do presente artigo. Do mesmo modo, buscou-se contato com autores internacionais para a aquisição de materiais não disponíveis em meio eletrônico, como capítulos de livros e artigos não indexados nas bases de dados consultadas. Também foi realizada uma extensa busca por informações complementares a partir das referências dos estudos utilizados.

\section{Cyberbullying - Definições e Estudos}

Diversos são os autores que buscam definir o fenômeno do cyberbullying (Agatston, Kowalski, \& Limber, 2007; Campbell, 2005; Hinduja \& Patchin, 2007, 2008, 2009; Kiriakidis \& Kavoura, 2010; Li, 2006; Mishna, Khoury-Kassabri, Gadalla, \& Daciuk, 2012; Smith, 2010). Contudo, não há consenso, ainda, em relação aos aspectos teóricos e também conceituais que apreendam o fenômeno em sua complexidade (Langos, 2012). Uma hipótese plausível para a ausência de uma definição precisa do cyberbullying diz respeito ao fato de que, na medida em que se observa uma significativa proliferação de novas tecnologias, emergem novos comportamentos e modos de agir diante de tais inovações (Spears, Slee, Owens, \& Johnson, 2009).

Em termos gerais, o processo de cyberbullying pode ser compreendido como um tipo específico de bullying que ocorre através de instrumentos tecnológicos e, sobretudo, telefones celulares e internet (Slonje \& Smith, 2008). Para Shariff (2011), é importante contextualizar o fenômeno, ou seja, considerando o tipo de ato realizado (por exemplo, violação de senhas, acesso e roubo de dados pessoais, piadas e comportamentos de humilhação, entre outros) e o meio onde ocorre (sites de redes sociais, e-mails, torpedos SMS [Short Message Service], entre outros). Tal perspectiva, de acordo com a autora, evita restringir o conceito de cyberbullying a uma visão simplista e reducionista, auxiliando também a precisar a etiologia e os respectivos 
impactos desses comportamentos praticados entre pares no ambiente virtual.

Outra definição amplamente aceita na literatura internacional é a de Hinduja e Patchin (2009). Estes autores descrevem o cyberbullying como um processo no qual alguém executa, proativa e repetidamente, atitudes como piadas acerca de uma pessoa em contextos virtuais ou quando um indivíduo "assedia alguém através de e-mails ou mensagens de texto ou ainda através de postagem de tópicos sobre assuntos que a vítima não aprecia" (Hinduja \& Patchin, 2009 , p. 48). A intencionalidade também é uma questão destacada pelos pesquisadores para que determinado comportamento seja caracterizado como cyberbullying e não apenas como uma brincadeira aleatória. $\mathrm{O}$ fenômeno consiste em uma relação que presume, pelo menos, dois papéis: vítima e agressor (Ortega et al., 2012). Entretanto, quando um adolescente exerce tanto um papel de agressor como de vítima, pode ser delineado um perfil que o caracteriza como vítima-agressor. Do mesmo modo como ocorre nas situações de bullying, os indivíduos que acompanham - ou assistem - aos episódios de agressão podem ser caracterizados como espectadores e, além disso, aqueles que por ventura se divertem ou mesmo compartilham os episódios podem ser compreendidos como apoiadores ou incentivadores do processo.

Muitos pesquisadores têm apontado aspectos que distinguem a vitimização entre pares que ocorre no contexto "real" (face a face) e o cyberbullying (Langos, 2012; Shariff, 2011; Smith, 2010). Nas agressões que ocorrem intramuros da escola, a vítima pode ser capaz de prever em quais situações se encontra em risco potencial (recreio, na ausência de figuras de autoridade, como professores e tutores, entre outros). Porém, quando a agressão ocorre por meios eletrônicos, escapar/evitar torna-se uma tarefa praticamente impossível (Smith, 2010). Isso ocorre uma vez que o agressor pode enviar mensagens para o aparelho celular ou para o e-mail da vítima, bem como lhe é possível, a qualquer hora ou momento do dia, postar vídeos e imagens constrangedoras em blogs, sites de relacionamento social, entre outros (Menesini et al., 2012).
Além disso, muitas vezes, as pessoas percebem o ciberespaço como impessoal, ou seja, como sendo um espaço no qual as ideias podem ser livremente expostas, ditas e compartilhadas ( $\mathrm{Li}, 2006)$. Na vida real, porém, a situação difere-se uma vez que o agressor pode limitar suas ações ou repensar cautelosamente antes de agir em função da reação da vítima ou do grupo de pares que presencia os atos agressivos, temendo a retaliação imediata (Shariff, 2011).

$\mathrm{Na}$ Tabela 1, apresentada a seguir, encontram-se sumarizados e discutidos os aspectos que distinguem a agressão entre pares por meio eletrônico daquela realizada face a face. Os critérios avaliados são os de agressividade, desequilíbrio de poder, audiência (espectadores), intencionalidade e repetição. A mesma tabela traz, ainda, informações e subsídios que suportam os motivos pelos quais o fenômeno do cyberbullying tem sido compreendido por um número cada vez maior de cientistas como uma forma peculiar e sem antecedentes de vitimização entre iguais, impactando severamente a vida de crianças e adolescentes em diversas partes do mundo e de modos cada vez mais diversificados.

Como se pode observar a partir da Tabela 1, o cyberbullying não é uma experiência face a face, ocorrendo sempre por meio da mediação de algum recurso tecnológico. Assim, possibilita ao agressor ficar anônimo, diferentemente da maior parte dos casos típicos de bullying (agressões físicas, insultos verbais, chantagem). Convém salientar que, em casos de bullying, os agressores também podem ficar anônimos utilizando, principalmente, formas indiretas de agressividade, como fofocas, espalhar rumores, denegrir a imagem de pessoas, dentre outros. Entretanto, percebe-se que, na maioria das vezes, os mesmos são identificados e têm seus comportamentos reforçados pelo grupo de pares. A opção pelo anonimato, nos casos de cyberbullying, pode ser compreendida a partir do chamado efeito da desinibição. Ou seja, as pessoas podem sentir confiança e coragem "diante da possibilidade de serem anônimas, 


\section{Tabela 1}

\section{Critérios Utilizados para Definição de Bullying x Cyberbullying}

\begin{tabular}{|c|c|}
\hline Critérios & Bullying \\
\hline Agressividade & $\begin{array}{l}\text { Pode ser expressa com uso de violência } \\
\text { física e insultos verbais. A vítima, em } \\
\text { geral, sabe qual é a pessoa ou o grupo } \\
\text { que lhe agride fisicamente. São comuns } \\
\text { casos extremos, como lutas corporais, } \\
\text { ferimentos sérios, entre outros Entretanto, } \\
\text { os agressores podem também não } \\
\text { ser conhecidos, uma vez que existem } \\
\text { agressividades de forma indireta como } \\
\text { fofocas, espalhar rumores, dentre outros } \\
\text { comportamentos deste tipo. } \dagger\end{array}$ \\
\hline
\end{tabular}

Desequilíbrio Um dos aspectos centrais do conceito de poder

Audiência

Intencionalidade Repetição de bullying e que o diferencia de outros comportamentos agressivos é o de que existe um desequilíbrio de poder entre agressor e vítima, sendo que este poder pode ser mantido através de ameaças e agressões físicas, sociais e/ou psicológicas $\dagger$

No processo de bullying, é comum que o grupo de pares reforce os atos dos agressores. Além disso, quando o processo ocorre em situações como recreio ou durante as aulas, os colegas presentes compõem a audiência. Esta passa a ser restrita e limitada ao contingente de estudantes que presenciam os atos

O bullying não se refere a um ato ocasional. $\mathrm{O}$ agressor apresenta a intenção de ferir, magoar ou humilhar a vítima $\dagger$

Um dos principais critérios para bullying é de que o fenômeno seja recorrente, sistemático; Há uma permanência, ao longo do tempo, das atitudes agressivas $\dagger$
Diferentemente do bullying, em que a situação de desequilíbrio de poder decorre em função de distinções como força, persuasão ou status, em sua manifestação eletrônica, o cyberbullying representa uma desvantagem em termos de habilidades tecnológicas (um usuário "mais avançado" pode vitimizar outra pessoa em decorrência de certos conhecimentos específicos). Além disso, o agressor pode recorrer ao anonimato, deixando a vítima sem possibilidades de identificar o autor das agressões e restringindo, assim suas formas de ação para combate desta violência *

No cyberbullying, todavia, a audiência pode ser nula (como nas situações em que o agressor dirige seus atos diretamente à vítima) ou infinita (como na postagem de vídeos, fotos ou outro material em sites e redes sociais)

Do mesmo modo, tal critério aplica-se ao cyberbullying, pois, ao enviar um e-mail ofensivo diretamente ou ainda ao postar uma foto indesejável da vítima em uma rede social constata-se que o ato é proativo, motivado

No cyberbullying, uma única ação realizada pode ser replicada inúmeras vezes por um número extenso de espectadores, não exigindo que o agressor repita o seu ato $\ddagger$ (ex: postar uma foto em um site ou um vídeo no Youtube, uma única vez, pode ser visualizado, salvo em um computador e compartilhado para uma ampla audiência) 
achando que nunca serão surpreendidas . . . e experimentam uma dificuldade maior em conter seus impulsos online do que em situações sociais no espaço real" (Palfrey \& Gasser, 2011, p. 108). Ainda em relação aos aspectos semelhantes entre os dois processos, pode-se inferir que o desequilíbrio de poder, a intenção de causar dano ou sofrimento e a repetição se encontram presentes. Todavia, nos atos de cyberbullying, o público pode chegar a um número muito mais expressivo de espectadores em comparação com os grupos pequenos que, normalmente, presenciam o bullying praticado na escola e/ou em pequenos grupos (Smith, 2010).

\section{Prevalência do Cyberbullying}

Quando um novo fenômeno emerge e ocupa grande parte das discussões da agen- da científica internacional, um dos primeiros movimentos que se observa é a busca por dados de prevalência. Com o cyberbullying não foi diferente. Cientistas de várias partes do mundo têm buscado mensurar a ocorrência dessa forma de vitimização entre pares e, do mesmo modo, compreender as associações entre cyberbullying e demais variáveis (Buelga, Cava, \& Musitu, 2010; Buelga \& Pons, 2012; Dehue, Bolman, \& Völlink, 2008; Di Lorenzo, 2012; Juvonen \& Gross, 2008; Li, 2006; Popovic-Citic, Djuric, \& Cvetkovic, 2011; Şahin, 2012; Wang et al., 2011; Williams \& Guerra, 2007). Nesse sentido, com o propósito de apresentar um panorama das referidas investigações sobre a prevalência do cyberbullying, organizou-se a Tabela 2. Os artigos foram arranjados levando-se em consideração o país no qual foi conduzido o estudo, a amostra, medidas

e instrumentos utilizados, dados de prevalência e principais achados.

\section{Tabela 2}

Estudos de Prevalência do Cyberbullying em Distintos Países

\begin{tabular}{|c|c|c|c|c|}
\hline Estudo & Origem & Amostra & Medidas utilizadas & Prevalência e demais achados \\
\hline Li (2006) & Canadá & $\begin{array}{l}264 \\
\text { adolescentes } \\
\text { regularmente } \\
\text { matriculados } \\
\text { nas séries } 7^{\mathrm{a}} \text {, } \\
8^{\mathrm{a}} \text { e } 9^{\mathrm{a}} . \text { A idade } \\
\text { média dos } \\
\text { participantes } \\
\text { não foi } \\
\text { informada. }\end{array}$ & $\begin{array}{l}\text { Um questionário anônimo } \\
\text { foi aplicado aos estudantes } \\
\text { contendo questões sobre a } \\
\text { frequência do uso de internet } \\
\text { e também da ocorrência de } \\
\text { cyberbullying. }\end{array}$ & $\begin{array}{l}\text { Os resultados mostraram que } 34 \% \text { dos } \\
\text { participantes relataram vitimização } \\
\text { "tradicional", e } 17 \% \text { afirmaram } \\
\text { terem realizado agressão por meio } \\
\text { da internet. A maioria dos estudantes } \\
\text { que foram vítimas de cyberbullying } \\
\text { ou que conheciam alguma vítima } \\
\text { afirmaram não terem informado este } \\
\text { fato a nenhum adulto. }\end{array}$ \\
\hline $\begin{array}{l}\text { Williams } \\
\& \text { Guerra } \\
(2007)\end{array}$ & $\begin{array}{l}\text { Estados } \\
\text { Unidos }\end{array}$ & $\begin{array}{l}3339 \\
\text { adolescentes } \\
\text { matriculados } \\
\text { nas séries } 5^{\mathrm{a}}, 8^{\mathrm{a}} \\
\text { e } 11^{\mathrm{a}} \text {. A idade } \\
\text { média dos } \\
\text { participantes } \\
\text { não foi } \\
\text { informada. }\end{array}$ & $\begin{array}{l}\text { Os pesquisadores aplicaram } \\
\text { questionários com vistas à } \\
\text { identificação da prevalência } \\
\text { de cyberbullying e também de } \\
\text { bullyingtradicional. Emambos } \\
\text { os casos, foram efetuadas } \\
\text { questões que mensurassem } \\
\text { tanto a o envolvimento como } \\
\text { perpetrador como também na } \\
\text { categoria de vítima. }\end{array}$ & $\begin{array}{l}\text { Apenas } 9,4 \% \text { dos participantes } \\
\text { declararam terem cometidos atos de } \\
\text { cyberbullying. Já um percentual de } \\
70,7 \% \text { de participantes afirmaram } \\
\text { terem praticado bullying verbal. } \\
\text { Os pesquisadores descobriram } \\
\text { que a percepção de clima escolar, } \\
\text { a aprovação moral para os } \\
\text { comportamentos agressivos e a } \\
\text { percepção de suporte entre o grupo } \\
\text { de iguais foram preditores de todas as } \\
\text { formas de vitimização experenciadas } \\
\text { pelos adolescentes do estudo. }\end{array}$ \\
\hline
\end{tabular}


Dehue et Holanda 1211

al. (2008) adolescentes

$(M=12,7$, $D P=0,73)$.
Um questionário sobre cyberbullying foi respondido pelos adolescentes e outro enviado aos pais. $70 \%$ dos adolescentes tiveram as respostas dos pais contabilizadas no estudo.

$23 \%$ dos participantes relataram vitimização por meio da internet. As formas mais comuns observadas de cyberbullying foram insultos $\mathrm{e}$ fofocas. Por outro lado, $16 \%$ dos adolescentes declararam terem praticado atos de cyberbullying. Em relação aos pais, constatou-se que $60 \%$ declararam o estabelecimento de regras claras sobre a frequência com a qual os filhos poderiam utilizar a internet.

Juvonen \& Gross (2008)

\section{Slonje} \& Smith (2008)

\begin{tabular}{|c|c|c|}
\hline Topçu, & Turquia & 183 \\
\hline Erdur- & & participantes, \\
\hline Baker, & & com idades \\
\hline \& Çapa- & & entre 14 a 15 \\
\hline Aydin & & $\operatorname{anos}(M=14,72$, \\
\hline (2008) & & $D P=0,42)$. \\
\hline
\end{tabular}
Unidos com idades entre 12 a 17 anos $(M=15,5$, $D P=1,47)$.

Suécia 360

participantes, com idades variando entre 12 a 20 anos. A idade média foi de 15,3 anos. O desvio-padrão informado no estudo. $D P=0,42)$.
Estados 1454 estudantes, Um questionário online da idade não foi interação entre pares na e anônimo foi aplicado, contendo questões sobre internet, via telefones celulares e também sobre a ocorrência de bullying.

Um questionário desenvolvido na Inglaterra foi traduzido para o uso na Suécia, contendo questões sobre distintas formas de vitimização online (ex: por e-mail, celular). Além disso, os participantes foram questionados sobre a percepção de gravidade do cyberbullying comparando com o impacto percebido do bullying tradicional.

Um questionário demográfico foi aplicado, contando com questões sobre hábitos na internet e também sobre o tempo gasto online pelos adolescentes. Além disso, um instrumento específico para mensurar a ocorrência de cyberbullying foi utilizado (Cyberbullying Inventory CBI), focando na ocorrência do fenômeno nos últimos seis meses.
$72 \%$ dos participantes relataram ao menos um episódio de vitimização online nos últimos 12 meses, sendo insultos e mensagens ameaçadoras as formas mais observadas. Além disso, $90 \%$ afirmaram que nenhum adulto foi comunicado acerca dos incidentes agressivos realizados através da internet.

$5,3 \%$ dos participantes relataram terem sido vítimas de cyberbullying, e $10,3 \%$ afirmaram terem cometido atos de agressão online. Além disso, observou-se que metade das vítimas de cyberbullying não relatou o fenômeno a ninguém, 35,7\% comentaram com um amigo e apenas $8,9 \%$ compartilharam a experiência com pais e ou responsáveis.

Em relação à vitimização online, os percentuais mais elevados foram verificados em estudantes de escolas privadas $(32,1 \%)$. Por outro lado, os percentuais mais elevados de agressão online (expulsar alguém de uma sala de bate-papo) foram verificados em estudantes de escolas públicas $(35,2 \%)$. 
Buelga et Espanha 2101

al. (2010)

Sourander

et al.

(2010)
Popovic- Sérvia 387

Citic et al. (2011)

Mishna et al. (2012)
Finlândia 2215

adolescentes, com idades variando entre 13 a 16 anos $(M=14,4$, $D P=1,1)$. participantes, com idades

entre 11 a 17 anos $(M=13,6$, $D P=1,30)$
Foi adaptado um questionário sobre vitimização entre pares investigando experiências relacionadas à ocorrência de cyberbullying via telefone celular e também através da internet (e-mail, ofensas em redes sociais, entre outros).

$\mathrm{O}$ estudo envolveu investigações tanto sobre declararam vitimização online experiências com vitimização nos últimos seis meses. Todavia, online nos últimos seis meses como também questões relacionadas a problemas psiquiátricos associados queixas psicossomáticas.

$24 \%$ dos participantes relataram vitimização através de telefones celulares, e aproximadamente um terço $(29 \%)$ via internet.

o percentual de participantes que declarou envolvimento enquanto agressor online foi superior: $7,4 \%$. $\mathrm{O}$ grupo de vítimas e agressores online foi de $5,4 \%$. Os adolescentes que relataram envolvimento com cyberbullying foram também os mais propensos a apresentarem psicopatologias e, do mesmo modo, queixas somáticas quando comparados ao grupo sem qualquer envolvimento com o fenômeno.

Foi construído um $10,06 \%$ dos participantes relataram participantes, com idades entre 11 a 15 anos $(M=13,2$ anos). O desviopadrão da idade não foi informado no estudo. questionário abordando três domínios. Um sobre informações demográficas (idade, sexo, escolaridade, etc), e outro relativo ao uso de internet e telefones celulares e, finalmente, dados sobre experiências com cyberbullying.

Canadá 2186 estudantes, Os pesquisadores utilizaram com idades entre 10 a 17 várias medidas com o objetivo de detectar fatores anos $(M=13,85$, $D P=1,98)$. de risco e proteção em relação ao cyberbullying. Assim, além de um instrumento voltado à identificação de comportamentos de cyber agressão e cyber vitimização, questões sobre envolvimento parental em relação ao uso de tecnologia por parte dos participantes e sobre experiências de violência na escola foram incluídas.
$23,8 \%$ dos participantes declararam vitimização online. Deste grupo, um percentual superior foi verificado nos estudantes do sexo feminino $(25,5 \%)$, contra $21,7 \%$ do sexo masculino. O percentual de estudantes que foram concomitantemente vítimas e perpetradores de cyberbullying representou mais de um quarto da $\operatorname{amostra}(25,7 \%)$. 
Observa-se que os estudos descritos na Tabela 2 priorizam o uso de instrumentos de autorrelato e algumas investigações incluíram, ainda, questionários aos pais e ou responsáveis dos adolescentes. Entretanto, os instrumentos existentes não dão conta de abarcar especificidades deste processo, tais como critérios de frequência dos atos agressivos online para definir a existência de um cyber agressor ou de uma cyber vítima. Percebe-se ainda que é comum a mensuração do cyberbullying considerando a forma de expressão, ou seja, via internet e telefones celulares, bem como comparando este fenômeno e seus impactos as já reportadas consequências associadas com o processo de bullying. Essa perspectiva de analisar os diferentes contextos (físico ou virtual), nos quais ocorrem e perpetuam as problemáticas de relacionamento entre pares, encontra respaldo teórico e empírico nos estudos atuais em Psicologia, principalmente envolvendo a Psicologia do Desenvolvimento (Sumter, Baumgartner, Valkenburg, \& Peter, 2012), mas sendo relevante também para área da pesquisa em Psicologia Clínica. O desenvolvimento de instrumentos para mensuração do cyberbullying, mais sensíveis à cultura latino-americana e, em específico, brasileira, é necessário e urgente. Ainda, questionários direcionados a pais, professores ou peer assessments (relato de pares) também podem ser alternativas interessantes para compreender a complexidade do fenômeno. Da mesma forma, pesquisas qualitativas que priorizem grupos focais e entrevistas abertas devem ser realizadas com maior frequência.

No tocante à prevalência, não se identifica um padrão entre os achados. Uma possível explicação para tais diferenças encontradas refere-se ao período de tempo que os autores dos estudos delimitaram para a investigação do cyberbullying - último mês, últimos seis meses, último ano, entre outros - e a faixa etária investigada. Outro aspecto que pode justificar resultados díspares diz respeito aos instrumentos utilizados em cada pesquisa, uma vez que medidas mais sensíveis podem detectar com maior acurácia as diferentes formas pelas quais o cyberbullying pode ocorrer (Garaigordobil, 2011; Lam \& Li, 2013). Ainda, os resultados podem ser influenciados pela dese- jabilidade social, dependendo dos procedimentos de coleta de dados e dos contextos nos quais os instrumentos foram aplicados. Os jovens podem temer retaliações das escolas e dos agressores, por exemplo, e não relatar seu envolvimento no cyberbullying.

\section{Cyberbullying: Impactos, Fatores de Risco e de Proteção}

Além de buscar verificar a prevalência do cyberbullying, estudos têm focado em questões relacionadas aos impactos e aos fatores de risco e proteção em relação ao fenômeno (Di Lorenzo, 2012; Heim, Brandtzæg, Endestad, Kaare, \& Torgersen, 2007; Mishna et al., 2012; Valcke, Bonte, De Wever, \& Rots, 2010; Valcke, Schellens, Van Keer, \& Gerarts, 2007). Diversas investigações associam o envolvimento no $c y$ berbullying com níveis elevados de sintomas de depressão (Baker \& Tanrıkulu, 2010; Hinduja \& Patchin, 2012; Mishna, Mclukie, \& Saini, 2009), isolamento social (Şahin, 2012), problemas na esfera educacional (Shariff, 2011), queixas somáticas (Sourander et al., 2010), entre outros.

Di Lorenzo (2012) discute um caso clínico de uma adolescente de 13 anos, vítima de $c y$ berbullying via mensagens de texto (torpedos SMS), que apresentava sintomas depressivos graves, culminando em uma tentativa de suicídio. Além disso, o pesquisador relatou a presença de sintomas relacionados ao Transtorno de Estresse Pós-Traumático na jovem, que também era vítima de bullying na escola. $\mathrm{O}$ autor apresenta hipóteses possíveis para a compreensão da vulnerabilidade da vítima, dentre as quais destaca o relativo descaso dos pais em relação ao sofrimento experienciado pela adolescente, além das características individuais apresentadas pela jovem, como timidez significativa e poucas relações de amizade e de baixa qualidade.

Outro fator pertinente para a análise de situações que possivelmente colocam os adolescentes em situação de risco foi destacado por Agatston et al. (2007). Os pesquisadores realizaram estudos de cunho qualitativo, por meio de grupos focais, com o intuito de compreenderem os motivos que sustentam a não comunicação aos pais 
e ou responsáveis sobre a ocorrência de vitimização online. Na visão dos adolescentes, o medo de perder certos privilégios, como o uso de telefones celulares e até mesmo a restrição do acesso à internet, são algumas das principais razões para que silenciem quando algum ato de cyberbullying ocorre. Além disso, os estudantes reportaram que não creem que os adultos, incluindo professores e pais, possam lhes ajudar no que tange a esse tipo peculiar de vitimização (Agatston et al., 2007). A falta de comunicação entre jovens, pais e professores com relação a estes aspectos da violência em meios virtuais constitui-se um preocupante fator de risco que deve ser alvo de intervenção de psicólogos e outros profissionais da área da saúde. Em determinadas culturas, como a brasileira, ainda impera um certo distanciamento em termos de conhecimentos acerca de tecnologias entre pais, filhos e, inclusive, entre professores e alunos. Estas diferenças, em termos de uso e conhecimento de tecnologias da informação e comunicação, podem deixar adultos e jovens afastados. Programas de intervenção direcionados a escolas podem incluir grupos com pais e atividades interativas que envolvam o uso de tecnologias e promovam trocas entre pais, professores e alunos. Suporte emocional e consciência acerca dos riscos, além dos benefícios do contexto virtual, na atualidade, devem ser focos de intervenções focais e preventivas.

Em relação aos fatores protetivos, estudos sugerem que filhos que experenciam adequado controle parental no que se refere ao uso da tecnologia apresentam também menos comportamentos de risco no ambiente virtual (Ayohama, Barnard-Brak, \& Talbert, 2011; Fleming, Greentree, Cocotti-Muller, Elias, \& Morrison, 2006; Heim et al., 2007; Mesch, 2009). Na medida em que os adolescentes se desenvolvem e adquirem cada vez maior independência com relação aos pais, estes costumam ajustar as práticas de supervisão, permitindo maior liberdade ao adolescente. Nesse sentido, a ênfase e atenção deve ser voltada à qualidade da relação estabelecida entre pais e filhos, bem como à coerência entre as práticas parentais utilizadas e a abertura para o diálogo e negociação (Stattin \& Kerr, 2000). Estas práticas parentais também devem ser res- significadas em consonância com o contexto sócio-histórico atual e suas especificidades.

O monitoramento parental, isto é, imposição de limite de tempo ou restrição de acesso a determinado(s) conteúdo(s) foi investigado e comparado ao uso da internet por parte dos filhos no estudo de Lee e Chae (2011). Esses autores encontraram associações significativas entre o tempo gasto na internet e uma diminuição da convivência familiar. Além disso, os autores verificaram que, quando os pais apresentavam práticas como "recomendar um site positivo ao filho" ou "navegar junto do filho", os filhos acabaram gastando mais tempo envolvidos em atividades educativas online e, portanto, menos propensos à experiência do cyberbullying. E, mesmo em tempos de casais com dupla carreira e pais que dispõem de pouco tempo para estar com seus filhos, enfatiza-se que a flexibilidade e a qualidade nas interações são características essenciais para a apromimação das famílias e para a garantia do efeito protetivo que pode advir desta convivência.

Conforme ressaltam Hinduja e Patchin (2009, p. 148), “o desenvolvimento de hábitos relacionados ao uso seguro de tecnologia deve ser estimulado desde muito cedo na vida das crianças na atualidade, de modo a garantir a efetiva internalizaçao desses comportamentos". Nesses casos, cabe ressaltar que a postura dos pais deve ser assertiva, envolvendo diálogo e negociação (Valcke et al., 2010). Ou seja, ao invés de os pais adotarem posturas intrusivas, tais como as referidas por Kowalski, Limber e Agatston (2012), envolvendo a checagem e leitura dos e-mails de seus filhos, é preferível a adoção de uma postura próxima, com adequada comunicação. Recomenda-se que sejam estabelecidas regras sobre o uso das tecnologias da informação e comunicação -TIC's - dentro de cada domicílio - consentimentos relativos à importância de reportarem aos pais quando na ocorrência de incidentes graves online, reserva de um espaço específico para o debate do uso da internet em casa, na escola e através de telefones celulares, entre outros. Por fim, enfatiza-se que cabe aos pais o fornecimento de instruções precisas sobre como agir caso seu filho envolva-se com cyber- 
bullying, bem como é importante que se informe às crianças e adolescentes que não apaguem os registros ou provas dos casos deste processo (Kowalski et al., 2012).

\section{Considerações Finais}

Não se pode negar, nos dias atuais, a influência das novas tecnologias da informação e comunicação. Vive-se em um mundo globalizado e virtual. Esse cenário contemporâneo apresenta inúmeras alternativas que se descortinam quando um sujeito é capaz de, em um único clique, entrar em contato e interagir com as mais diversas possibilidades de expressão de opiniões, sentimentos e desejos. Assim, já é relativamente consenso que as crianças e adolescentes, hoje, se desenvolvem com uma consciência global, sendo tal movimento impulsionado pelas TIC's. Com efeito, os jovens nascidos em um mundo permeado por estas tecnologias são chamados de nativos digitais (Palfrey \& Gasser, 2011). A interação dos indivíduos com estas novas tecnologias da informação e comunicação transformou inúmeros aspectos do relacionamento interpessoal e, por consequência, dos processos de representação da identidade, formas de aprendizagem e de relacionamento (Arnett, 2002).

$\mathrm{O}$ entendimento sobre as influências do "mundo virtual" no desenvolvimento humano ainda necessita ser aprofundado. Observa-se que o uso e contato com as TIC's não se restringe ou afeta jovens de uma cultura ou nível socioeconômico específico e tampouco se limita a uma faixa etária - infância, pré-adolescência ou adolescência. Muito se discute também com relação aos riscos e vantagens destes novos recursos. $\mathrm{Ou}$ seja, o uso de muitos recursos tecnológicos ao mesmo tempo (media multitasking) pode ser benéfico e estimulante aos jovens, mas, por outro lado, pode gerar irritação, baixa produtividade, aumento de ansiedade e problemas atencionais (Uhls, Espinoza, Greenfield, Subrahmanyam, \& Šmahel, 2011). No que se refere aos comportamentos agressivos, como é possível inferir através dos estudos anteriormente descritos, o processo não é diferente e ainda necessita ser mais estudado.
O cyberbullying pode ocorrer em paralelo ao processo do bullying, razão pela qual diversas nações têm se empenhado na elaboração de políticas focadas especificamente no combate destes dois fenômenos em conjunto (Raskauskas, \& Stoltz, 2007; Smith, Smith, et al., 2008). Assim como as diferentes formas de interação interpessoal no contexto virtual, ainda se desconhece todas as características do cyberbullying, suas consequências em curto, médio e longo prazo, bem como o impacto nas crenças e comportamentos dos jovens (Olweus, 2012).

A revisão de estudos realizada permite inferir que se trata de um fenômeno que pode acarretar em sérios prejuízos sociais, emocionais e cognitivos aos envolvidos, principalmente pelo seu caráter atemporal e pela magnitude de seu alcance. Uma vítima de cyberbullying, em geral, não tem para onde ir e se esquivar dessa violência. O cyberbullying constitui "uma realidade da era digital" (Langos, 2012, p. 285) que, de acordo com Smith (2010), é um fenômeno decorrente ou relacionado às diversas transformações que ocorreram no século XXI.

Resultado ou consequência do avanço das TIC's, o cyberbullying é especialmente frequente entre crianças e adolescentes. Isso ocorre porque os nativos digitais (Palfrey \& Gasser, 2011) são usuários ávidos e familiarizados com as tecnologias e internet. Entretanto, os mesmos não possuem plenas condições de distinguir entre aquilo que a tecnologia pode representar de positivo do que traduz-se como risco ( $\mathrm{Li}, 2006)$. As pesquisas sobre cyberbullying sugerem que os jovens têm dificuldades de adequadamente dimensionar as reais consequências de seus atos agressivos e é possível pensar que estas dificuldades sejam mais acentuadas e graves que nos casos de bullying. Investigações enfatizam o papel do monitoramento parental, das práticas educativas saudáveis, intervenções clínicas que possam psicoeducar adequadamente com relação ao fenômeno, além de estímulos variados para uma vinculação ajustada entre pais e filhos (Hinduja \& Patchin, 2009; Lee \& Chae, 2011; Valcke et al., 2010).

Pode não ser novidade que a supervisão e limites dos pais favoreçam o desenvolvimento saudável dos filhos, mas diante desse novo ce- 
nário virtual constata-se que os pais necessitam de informações sobre o cyberbullying e TIC's de um modo geral. Assim, estes pais estarão mais capacitados para auxiliar, efetivamente, seus filhos na resolução de conflitos e na prevenção de eventos adversos neste cenário que possam impactar e prejudicar o desenvolvimento saudável de crianças e adolescentes. Governo e empresas que atuam no ramo de tecnologia compartilham igualmente a responsabilidade em prover ações que garantam segurança ao uso das mais variadas TIC's por parte de populações mais vulneráveis (Palfrey \& Gasser, 2011).

No que diz respeito à Psicologia e, em especial à Psicologia Clínica, torna-se importante compreender os fatores de risco e proteção para a ocorrência do cyberbullying, assim como crenças e comportamentos relacionados a este novo fenômeno, visando o desenvolvimento de alternativas para a prevenção e intervenção psicológica (Patchin \& Hinduja, 2010; Wendt \& Lisboa, 2013). É importante salientar que identificar fatores de risco e proteção implica em uma análise aprofundada dos processos de desenvolvimento cognitivo e social dos jovens na atualidade. Investigações enfatizam que o $c y$ berbullying pode prejudicar ou mesmo interromper o curso de desenvolvimento normativo dos jovens, como em casos de suicídio relacionados à experiência de vitimização online (Hinduja \& Patchin, 2010, 2012). Ademais, pesquisas que constataram a associação entre o cyberbullying e níveis elevados de sintomas de depressão e ansiedade reforçam a ideia de que experenciar o fenômeno, tanto como vítima como na qualidade de agressor, pode impactar negativamente o curso desenvolvimental (Baker \& Tanrikulu, 2010; Sourander et al., 2010).

Em um futuro próximo, muitos questionamentos ainda surgirão sobre esta temática, pois, na medida em que as TIC's avançam e adentram mais e mais na vida dos indivíduos e nas instituições sociais, novas modalidades de interação, desenvolvimento e, por conseguinte, de agressão entre pares podem surgir. Assim, não se pretende esgotar o assunto, mas o presente estudo teve, primordialmente, a intenção de alertar e debater criticamente para a complexidade do processo de cyberbullying na atualidade. Abordagens focadas em investigar efeitos das tecnologias nos comportamentos dos jovens precisam ser substituídas por abordagens construtivistas e assertivas, que questionem e investiguem sobre os contextos de interação que os jovens vêm optando para si mesmos. Acredita-se que, na medida em que pais, educadores, responsáveis pela elaboração de políticas públicas voltadas para crianças e adolescentes e a sociedade de um modo geral tiverem adequada consciência dos aspectos positivos e negativos do uso das TIC's pelos mais jovens, assim como acerca do cyberbullying, atitudes preventivas e intervenções mais eficicazes podem ser propostas.

\section{Referências}

Agatston, P. W., Kowalski, R., \& Limber, S. (2007). Students' perspectives on Cyber Bullying. Journal of Adolescent Health, 41(6), S59-S60. doi:10.1016/j.jadohealth.2007.09.003

Anderson, C. A., \& Bushman, B. J. (2002). Human aggression. Annual Review of Psychology, 53(1), 27-51. doi:10.1146/annurev. psych.53.100901.135231

Arnett, J. J. (2002). The Psychology of Globalization. American Psychologist, 57(10), 774-783. doi:10.1037/0003-066X.57.10.774

Ayohama, I., Barnard-Brak, L., \& Talbert, T. (2011). Cyberbullying among high school students: Cluster analysis of sex and age differences and the level of parental monitoring. International Journal of Cyber Behavior, Psychology and Learning, 1(1), 25-35. doi:10.4018/ijcyberbullyingpl.2011010103

Baker, Ö. E., \& Tanrıkulu, İ. (2010). Psychological consequences of Cyber Bullying experiences among Turkish secondary school children. Procedia - Social and Behavioral Sciences, 2(2), 2771-2776. doi:10.1016/j.sbspro.2010.03.413

Berger, C., \& Lisboa, C. (2009). Violencia escolar: estúdios y posibilidades de intervención em Latinoamérica. Santiago, Chile: Editorial Universitária.

Binsfeld, A. R., \& Lisboa, C. (2010). Bullying: Um estudo sobre papéis sociais, ansiedade e depressão no contexto escolar do Sul do Brasil. Interpersona, 4(1), 74-105. 
Buelga, S., Cava, M., \& Musitu, G. (2010). Cyberbullying: victimización entre adolescentes a través del teléfono móvil y de Internet. Psicothema, 22(4), 784-789.

Buelga, S., \& Pons, J. (2012). Agresiones entre adolescentes a través del teléfono móvil y de internet. Psychosocial Intervention, 21(1), 91-101. doi:10.5093/in2012v21n1a2

Campbell, M. A. (2005). Cyber Bullying: An old problem in a new guise? Australian Journal of Guidance and Counselling, 15(1), 68-76. doi:10.1375/ajgc.15.1.68

Couvillon, M. A., \& Ilieva, V. (2011). Recommended practices: A Review of Schoolwide Preventative Programs and Strategies on Cyberbullying. Preventing School Failure, 55(2), 96-101. doi:10.1 080/1045988X.2011.539461

Dehue, F., Bolman, C., \& Völlink, T. (2008). Cyberbullying: Youngsters' experiences and parental perception. CyberPsychology \& Behavior, 11(2), 217-223. doi:10.1089/cpb.2007.0008

DeWall, C. N., Anderson, C. A., \& Bushman, B. J. (2011). The General Aggression Model: Theoretical extensions to Violence. Psychology of Violence, 1(3), 245-258. doi:10.1037/a0023842

Di Lorenzo, M. (2012). New forms of violence among peers: From Bullying to Cyberbullying. Revista Médica Uruguaia, 28(1), 48-53.

Dodge, K. A., Bates, J. E., \& Pettit, G. S. (1990). Mechanisms in the Cycle of Violence. Science, 250, 1678-1683.

Dooley, J. J., Pyżalski, J., \& Cross, D. (2009). Cyberbullying versus Face-to-Face Bullying. Zeitschrift für Psychologie / Journal of Psychology, 217(4), 182-188. doi:10.1027/00443409.217.4.182

Fleming, M. J., Greentree, S., Cocotti-Muller, D., Elias, K. A., \& Morrison, S. (2006). Safety in Cyberspace - Adolescents' safety and exposure online. Youth and Society, 38(2), 135-154.

Garaigordobil, M. (2011). Prevalencia y consecuencias del Cyberbullying: una revisión. International Journal of Psychology and Psychological Therapy, 11(2), 233-254.

Hansen, T. B., Steenberg, L. M., Palic, S., \& Elklit, A. (2012). A review of psychological factors related to Bullying victimization in schools. $A g-$ gression and Violent Behavior, 17(4), 383-387. doi:10.1016/j.avb.2012.03.008
Heim, J., Brandtzæg, P. B., Endestad, T., Kaare, B. H., \& Torgersen, L. (2007). Children's usage of media technologies and psychosocial factors. New Media \& Society, 9(3), 425-454.

Hinduja, S., \& Patchin, J. W. (2007). Offline consequences of online victimization: School violence and delinquency. Journal of School Violence, 6(3), 89-112. doi:10.1300/J202v06n03_06

Hinduja, S., \& Patchin, J. W. (2008). Cyberbullying: An exploratory analysis of factors related to offending and victimization. Deviant Behavior, 29(2), 129-156. doi:10.1080/01639620701457816

Hinduja, S., \& Patchin, J. W. (2009). Bullying beyond the schoolyard: Preventing and responding to Cyberbullying. Thousand Oaks, CA: Corwin Press.

Hinduja, S., \& Patchin, J. W. (2010). Bullying, Cyberbullying, and Suicide. Archives of Suicide Research, 14(3), 206-221. doi:10.1080/138111 18.2010 .494133

Hinduja, S., \& Patchin, J. W. (2012). Cyberbullying may exacerbate problems that can lead to suicide. In L. Gerdes (Ed.), Cyberbullying (pp. 5256). New York: Cengage Learning.

Hong, J. S., \& Espelage, D. L. (2012). A review of research on bullying and peer victimization in school: An ecological system analysis. Aggression and Violent Behavior, 17(4), 311-322. doi:10.1016/j.avb.2012.03.003

Juvonen, J., \& Gross, E. F. (2008). Extending the School Grounds? Bullying experiences in cyberspace. Journal of School Health, 78(9), 496-505. doi:10.1111/j.1746-1561.2008.00335.x

Kandersteg Declaration Against Bullying in Children and Youth. (2007). Declaração de Kandersteg Anti-Vitimização Entre Crianças e Jovens. Recuperado em 29 de julho de 2012 de http:// www.kanderstegdeclaration.com/storage/Portugiesisch.pdf

Kiriakidis, S. P., \& Kavoura, A. (2010). Cyberbullying: A review of the literature on harassment through the internet and other electronic means. Family and Community Health, 33(2), 82-93. doi:10.1097/FCH.0b013e3181d593e4

Kowalski, R., Limber, S., \& Agatston, P. W. (2012). Cyberbullying: Bullying in the digital age. Malden, MA: Wiley-Blackwell

Lam, L. T., \& Li, Y. (2013). The validation of the E-Victimisation Scale (E-VS) and the E-Bul- 
lying Scale (E-BS) for adolescents. Computers in Human Behavior, 29(1), 3-7. doi:10.1016/j. chb.2012.06.021

Langos, C. (2012). Cyberbullying: The challenge to define. Cyberpsychology, Behavior, and Social Networking, 15(6), 285-289. doi:10.1089/cyber.2011.0588

Law, D. M., Shapka, J. D., Hymel, S., Olson, B. F., \& Waterhouse, T. (2012). The changing face of bullying: An empirical comparison between Traditional and Internet Bullying and Victimization. Computers in Human Behavior, 28(1), 226-232. doi:10.1016/j.chb.2011.09.004

Lee, S. J., \& Chae, Y. G. (2011). Children's internet use in a family context: Influence on family relationships and parental mediation. CyberPsychology \& Behavior, 10(5), 640-644. doi:10.1089/ cpb.2007.9975

Li, Q. (2006). Cyberbullying in Schools: A research of gender differences. School Psychology International, 27(2), 157-170. doi:10.1177/0143034306064547

Lisboa, C., Braga, L., \& Ebert, G. (2009). O fenômeno bullying ou vitimização entre pares na atualidade: Definições, formas de manifestação e possibilidades de intervenção. Contextos Clínicos, 2(1), 59-71. doi:10.4013/ctc.2009.21.07

Little, T. D., Henrich, C. C., Jones, S. M., \& Hawley, P. H. (2003). Disentangling the 'whys' from the 'whats' of aggressive behavior. International Journal of Behavioral Development, 27, 122133.

Malta, D. C., Silva, M., Mello, F. C., Monteiro, R. A., Sardinha, L. M, Crespo, C., ...Porto, D. N. (2010). Bullying nas escolas brasileiras: Resultados da Pesquisa Nacional de Saúde do Escolar (PeNSE), 2009. Ciência \& Saúde Coletiva, 15(2), 3065-3076.

Menesini, E., Nocentini, A., Palladino, B. E., Frisén, A., Berne, S., Ortega-Ruiz, R., Calmaestra, J., ...Smith, P. K. (2012). Cyberbullying definition among adolescents: A comparison across six European countries. Cyberpsychology, Behavior, and Social Networking, 15(9), 455-463. doi:10.1089/cyber.2012.0040

Mesch, G. S. (2009). Parental mediation, online activities, and Cyberbullying. CyberPsychology \& Behavior, 12(4), 387-393. doi:10.1089/ cpb.2009.0068
Mishna, F., Khoury-Kassabri, M., Gadalla, T., \& Daciuk, J. (2012). Risk factors for involvement in Cyber Bullying: Victims, bullies and bully-victims. Children and Youth Services Review, 34(1), 63-70. doi:10.1016/j.childyouth.2011.08.032

Mishna, F., Mclukie, A., \& Saini, M. (2009). Realworld dangers in an online reality: A qualitative study examining online relationships and cyber abuse. Social Work Research, 33, 107-118.

Olweus, D. (1993). Bullying at school: What we know and what we can do. London: Blackwell.

Olweus, D. (2012). Cyberbullying: An overrated phenomenon? European Journal of Developmental Psychology, 9(5), 1-19. doi:10.1080/17405629. 2012.682358

Ortega, R., Elipe, P., Mora-Merchán, J. A., Genta, M. L., Brighi, A., Guarini, A., Smith, P. K., ...Tippett, N. (2012). The emotional impact of Bullying and Cyberbullying on victims: A European Cross-National Study. Aggressive Behavior, 38, 342-356. doi:10.1002/ab.21440

Palfrey, J., \& Gasser, U. (2011). Nascidos na Era Digital: Entendendo a primeira geração de Nativos Digitais. Porto Alegre, RS: ArtMed.

Patchin, J. W., \& Hinduja, S. (2010). Cyberbullying and self-esteem. Journal of School Health, 80(12), 614-621.

Popovic-Citic, B., Djuric, S., \& Cvetkovic, V. (2011). The prevalence of Cyberbullying among adolescents: A case study of middle schools in Serbia. School Psychology International, 32(4), 412424. doi:10.1177/0143034311401700

Raskauskas, J., \& Stoltz, A. D. (2007). Involvement in traditional and electronic bullying among adolescents. Developmental Psychology, 43(3), 564-575. doi:10.1037/0012-1649.43.3.564

Rigby, K. (2004). Addressing Bullying in Schools: Theoretical perspectives and their implications. School Psychology International, 25, 287-300. doi:10.1177/0143034304046902

Şahin, M. (2012). The relationship between the cyberbullying/cybervictmization and loneliness among adolescents. Children and Youth Services Review, 34(4), 834-837. doi:10.1016/j.childyouth.2012.01.010

Shariff, S. (2011). Ciberbullying: Questões e soluções para a escola, a sala de aula e a família. Porto Alegre, RS: ArtMed. 
Slonje, R., \& Smith, P. K. (2008). Cyberbullying: Another main type of bullying? Scandinavian Journal of Psychology, 49(2), 147-154. doi:10.1111/ j.1467-9450.2007.00611.x

Smith, P. K. (2010). Cyberbullying: The European perspective. In J. Mora-Merchan \& T. Jaeger (Eds.), Cyberbullying: A cross-national comparison (pp. 7-19). Landau, Germany: Verlag Emprische Padagogik.

Smith, P. K. (2012). Cyberbullying and Cyber Aggression. In S. R. Jimerson, A. B. Nickerson, M. J. Mayer, \& M. J. Furlong (Eds.), Handbook of School Violence and School Safety: International Research and Practice (pp. 93-103). New York: Routledge.

Smith, P. K., Mahdavi, J., Carvalho, M., Fisher, S., Russell, S., \& Tippett, N. (2008). Cyberbullying: Its nature and impact in secondary school pupils. Journal of Child Psychology and Psychiatry, 49(4), 376-385.

Smith, P. K., Smith, C., Osborn, R., \& Samara, M. (2008). A content analysis of school Anti-Bullying policies: Progress and limitations. Educational Psychology in Practice, 24(1), $1-12$.

Sourander, A., Klomek, A. B., Ikonen, M., Lindroos, J., Luntamo, T., Koskelainen, M., ...Helenius, H. (2010). Psychosocial risk factors associated with Cyberbullying among adolescents: A Population-Based Study. Archives of General Psychiatry, 67(7), 720-728. doi:10.1001/archgenpsychiatry.2010.79

Spears, B., Slee, P., Owens, L., \& Johnson, B. (2009). Behind the scenes and screens. Zeitschrift für Psychologie / Journal of Psychology, 217(4), 189-196. doi:10.1027/0044-3409.217.4.189

Stattin, H., \& Kerr, M. (2000). Parental monitoring: A reinterpretation. Child Development, 71, 1072-85.

Sumter, S. R., Baumgartner, S. E., Valkenburg, P. M., \& Peter, J. (2012). Developmental trajectories of peer victimization: Off-line and online experiences during adolescence. Journal of $\mathrm{Ad}$ olescent Health, 50(6), 607-613. doi:10.1016/j. jadohealth.2011.10.251

Topçu, Ç., Erdur-Baker, Ö., \& Çapa-Aydin, Y. (2008). Examination of Cyberbullying experiences among Turkish students from different school types. CyberPsychology \& Behavior, 11(6), 643-648.
Uhls, Y. T., Espinoza, G., Greenfield, P., Subrahmanyam, K., \& Šmahel, D. (2011). Internet and other Interactive Media. In B. Brown \& M. Prinstein (Eds.), Encyclopedia of Adolescence (pp. 160-168). San Diego, CA: Academic Press.

Valcke, M., Bonte, S., De Wever, B., \& Rots, I. (2010). Internet parenting styles and the impact on internet use of primary school children. Computers \& Education, 55(2), 454-464. doi:10.1016/j.compedu.2010.02.009

Valcke, M., Schellens, T., Van Keer, H., \& Gerarts, M. (2007). Primary school children's safe and unsafe use of the Internet at home and at school: An exploratory study. Computers in Human Behavior, 23, 2838-2850.

Wang, J., Nansel, T. R., \& Iannotti, R. J. (2011). Cyber and traditional Bullying: Differential association with depression. The Journal of Adolescent Health, 48(4), 415-417.

Wendt, G. W., Campos, D. M., \& Lisboa, C. (2010). Agressão entre pares e vitimização no contexto escolar: Bullying, Cyberbullying e os desafios para a educação contemporânea. Cadernos de Psicopedagogia, 8, 41-52.

Wendt, G. W., \& Lisboa, C. S. de M. (2013). Agressão entre pares no espaço virtual: definições, impactos e desafios do cyberbullying. Psicologia Clínica, 25(1), 73-87. doi:10.1590/S010356652013000100005

Williams, K., \& Guerra, N. (2007). Prevalence and predictors of Internet Bullying. Journal of Adolescent Health, 41(6), S14-S21.

Ybarra, M. L. (2004). Linkages between Depressive Symptomatology and Internet harassment among young regular internet users. CyberPsychology and Behavior, 7, 247-257.

Ybarra, M. L., Boyd, D., Korchmaros, J. D., \& Oppenheim, J. (2012). Defining and Measuring Cyberbullying within the Larger Context of Bullying Victimization. Journal of Adolescent Health, 51(1), 53-58. doi:10.1016/j.jadohealth. 2011.12 .031 